\title{
Effects of the Once-Weekly DPP4 Inhibitor Omarigliptin on Glycemic Control in Patients with Type 2 Diabetes Mellitus on Maintenance Hemodialysis: A 24-Week Open-Label, Multicenter Randomized Controlled Study
}

\author{
Yuta Yoshizawa · Michihiro Hosojima (D) - Hideyuki Kabasawa · Naohito Tanabe • \\ Daisuke Ugamura · Yutaka Koda · Hisaki Shimada · Tetsuya Takasawa • Takahito Ito • \\ Tadahiro Kitamura · Masaki Kobayashi · Yoshiki Suzuki · Ichiei Narita · Akihiko Saito \\ Received: November 16, 2020 / Accepted: December 19, 2020 / Published online: January 20, 2021 \\ (c) The Author(s) 2021
}

\section{ABSTRACT}

Introduction: Dipeptidyl peptidase 4 (DPP4) inhibitors are widely used in patients with type 2 diabetes mellitus (T2DM) on maintenance hemodialysis (HD), but the efficacy of the

Supplementary Information The online version contains supplementary material available at https:// doi.org/10.1007/s13300-020-00991-y.

Y. Yoshizawa · D. Ugamura · A. Saito Department of Applied Molecular Medicine, Kidney Research Center, Niigata University Graduate School of Medical and Dental Sciences, 1-757 Asahimachi-dori, Chuo-ku, Niigata, Niigata 9518510, Japan

Y. Yoshizawa · D. Ugamura · I. Narita Department of Clinical Nephrology and Rheumatology, Kidney Research Center, Niigata University Graduate School of Medical and Dental Sciences, 1-757 Asahimachi-dori, Chuo-ku, Niigata, Niigata 951-8510, Japan

M. Hosojima $(\bowtie) \cdot$ H. Kabasawa

Department of Clinical Nutrition Science, Kidney

Research Center, Niigata University Graduate

School of Medical and Dental Sciences, 1-757

Asahimachi-dori, Chuo-ku, Niigata, Niigata 951-

8510, Japan

e-mail: hoso9582@med.niigata-u.ac.jp

N. Tanabe

Health and Nutrition, University of Niigata

Prefecture, 471 Ebigase, Higashi-ku, Niigata, Niigata

950-8680, Japan once-weekly DPP4 inhibitor omarigliptin is not known.

Methods: This prospective, randomized, openlabel, parallel-group, non-inferiority/superiority, once-daily DPP4 inhibitor linagliptin-controlled, multicenter study examined glycemic control and safety of omarigliptin (UMIN000024284). Sample size was calculated to confirm non-inferiority in terms of changes in glycated hemoglobin (HbA1c). We enrolled

\section{Y. Koda}

Koda Internal Medicine Clinic, 3748 Yoshida,

Tsubame, Niigata 959-0264, Japan

\section{H. Shimada - T. Takasawa}

Shinrakuen Hospital, 3-3-11 Shin-dori-minami, Nishi-ku, Niigata, Niigata 950-2087, Japan

\section{T. Ito}

Kataguilli Medical Center, 4-3-9 Sumiyoshi-cho, Shibata, Niigata 957-0061, Japan

T. Kitamura · M. Kobayashi

Metabolic Signal Research Center, Institute for Molecular and Cellular Regulation, Gunma University, 3-39-15 Showa-machi, Maebashi, Gunma 371-8512, Japan

\section{Y. Suzuki}

Health Administration Center, Niigata University, 8050, Ikarashi 2-no-cho, Nishi-ku, Niigata, Niigata 950-2181, Japan 
33 patients with T2DM on maintenance HD who had been treated with linagliptin for at least 3 months. The patients were randomized to receive omarigliptin $(12.5 \mathrm{mg} /$ week; $n=16)$ or linagliptin (5 mg/day; $n=17)$. Primary endpoints were changes in HbA1c and glycoalbumin (GA) over 24 weeks.

Results: Differences in the mean change in primary endpoint values between the omarigliptin and linagliptin groups were $-0.61 \%[-1.14$, - 0.09] for HbA1c, with a two-tailed upper 95\% limit (i.e., one-tailed $97.5 \%$ upper limit) of $0.25 \%$, below the non-inferiority limit, and $-1.67 \%$ $[-4.23,+0.88]$ for GA, with a two-tailed upper $95 \%$ limit of $0.75 \%$, above the non-inferiority limit. At 24 weeks, the omarigliptin group showed significantly greater reduction in HbA1c than the linagliptin group $(-0.2 \% \pm 0.6 \% \quad$ vs. $0.4 \% \pm 0.8 \%$, two-tailed $p=0.024)$ and significantly greater reduction in blood glucose after a single HD session $(-18.4 \pm 31.4 \mathrm{mg} / \mathrm{dL}$ vs. $25.2 \pm 59.5 \mathrm{mg} / \mathrm{dL}$, respectively, two-tailed $p=0.019$ ). No subjects in the omarigliptin group developed hypoglycemia.

Conclusions: Our data showed that omarigliptin was non-inferior to linagliptin in glycemic control. Omarigliptin is feasible for glycemic control in patients with T2DM on maintenance HD.

Clinical Trials Registration: UMIN000024284.

Keywords: Linagliptin; Hemodialysis; Onceweekly dipeptidase 4 inhibitor; Omarigliptin; Type 2 diabetes mellitus

\section{Key Summary Points}

Why carry out this study?

Efficacy of the once-weekly DPP4 inhibitor omarigliptin is unknown in patients with type 2 diabetes mellitus on maintenance hemodialysis.

There are no reports of comparisons between once-weekly and once-daily DPP4 inhibitors in glycemic control and treatment satisfaction in patients with type 2 diabetes mellitus on maintenance hemodialysis.

\section{What was learned from the study?}

Once-weekly DPP4 inhibitor omarigliptin was non-inferior to once-daily DPP4 inhibitor linagliptin in its glycemic control.

If patients on hemodialysis can take onceweekly drugs, such as omarigliptin, at a hemodialysis center, they might maintain adherence almost perfectly.

Once-weekly DPP4 inhibitor omarigliptin is feasible for glycemic control in patients with type 2 diabetes mellitus on maintenance hemodialysis.

\section{DIGITAL FEATURES}

This article is published with digital features, including a summary slide, to facilitate understanding of the article. To view digital features for this article go to https://doi.org/10.6084/ m9.figshare.13417397.

\section{INTRODUCTION}

Insulin injection therapy is mainly used for glycemic control in patients on maintenance hemodialysis (HD) with type 2 diabetes mellitus (T2DM). Some problems arise in insulin therapy though, such as hypoglycemia, cumbersomeness, and pain from the injection itself [1]. Patients on maintenance HD endure repeated venipuncture for venous access at each HD session (usually three times a week) and also need daily injections of insulin. It would be ideal to achieve good glycemic control without insulin injection by using only oral hypoglycemic agents (OHAs), but the use of available OHAs is limited in these patients because of the risk of prolonged hypoglycemia.

Dipeptidyl peptidase 4 (DPP4) inhibitors have the benefits of not only providing proper glycemic control but also being associated with a lower incidence of hypoglycemia and good safety, even in patients with chronic kidney disease [2], so they are widely used in patients 
on maintenance HD [3]. Once-weekly DPP4 inhibitors recently became commercially available, and compared with once-daily DPP4 inhibitors, they are not inferior in terms of glycemic control and their efficacy has been reported [4]. The once-weekly DPP4 inhibitor omarigliptin is reported to be non-inferior to other OHAs and to have improved adherence in patients with T2DM [5]. Omarigliptin is also an option for patients with severe renal dysfunction [6]. Because patients on maintenance HD need to take many oral medications and often have problems with adherence $[7,8]$, it is likely that omarigliptin could improve their adherence.

However, no reports have compared onceweekly and once-daily DPP4 inhibitors in terms of changes in glycemic control in patients with T2DM on maintenance HD. Therefore, in this study, we investigated the non-inferiority of once-weekly omarigliptin compared with the once-daily DPP4 inhibitor linagliptin in these patients. We also studied the efficacy of omarigliptin in association with changes in plasma glucagon and active glucagon-like peptide 1 (GLP-1) levels and treatment satisfaction.

\section{METHODS}

\section{Participants}

Eligible patients (1) had been on maintenance HD for more than 6 months, (2) were aged at least 20 years with T2DM treated using DPP4 inhibitors for more than 3 months, and (3) had given written informed consent for the use of their clinical data in this study. Exclusion criteria were (1) treatment with GLP-1 receptor agonists, (2) hypersensitivity to DPP4 inhibitors or GLP-1 receptor agonists, (3) severe diabetic ketosis, coma, or pre-coma, (4) severe active infection, severe trauma, or in the perioperative period, (5) severe heart or liver dysfunction, (6) other conditions such as pituitary gland or adrenal gland dysfunction, impaired nutrition, starvation, irregular or insufficient dietary intake, hyposthenia, excessive muscular exercise, or heavy alcohol consumption, (7) pregnant, breastfeeding, or may be pregnant, (8) uncontrolled hyperglycemia (glycated hemoglobin $[\mathrm{HbA} 1 \mathrm{c}] \geq 9 \%$ or glycoalbumin $[\mathrm{GA}]$ $\geq 27 \%$ ) on current treatment with OHAs, or (9) considered ineligible for this trial by the attending physician for any medical reasons.

\section{Study Design}

This was a prospective, randomized, open-label, parallel-group, non-inferiority/superiority, once-daily linagliptin-controlled, multicenter study conducted at Niigata University Medical and Dental Hospital and three affiliated dialysis facilities between April 2017 and March 2018 (UMIN000024284). This study was approved by the Ethics Committee of Niigata University (approval number 2015-1277).

The overall study design is shown in Fig. 1. After providing written informed consent to participate, patients who had been taking linagliptin continued as they were, but those who had been taking other DPP4 inhibitors changed to linagliptin. We provided a pre-observation period of at least 3 months and checked for adverse events or side effects through medical interviews and by investigating standard laboratory parameters. We then randomized the patients into a group that continued linagliptin and a group that switched to omarigliptin after the pre-observation period. Patients in the linagliptin group took one tablet daily after breakfast ( $5 \mathrm{mg} /$ day), whereas patients in the omarigliptin group took one tablet every first HD day of the week at their HD center $(12.5 \mathrm{mg} /$ week). The nurses confirmed that the patients in the omarigliptin group took a tablet at their HD center and instructed the patients in the linagliptin group to take a tablet every day.

For randomization, we used the sealed envelope method. The random sequence of envelope allocations was generated using block randomization. The block sequence was determined on the basis of random numbers generated in $\mathrm{Excel}^{\mathrm{TM}}$. A controller outside the trial administration center performed the randomization process and created the sealed envelopes.

The observation period was 24 weeks. From the start of the observation period, no 


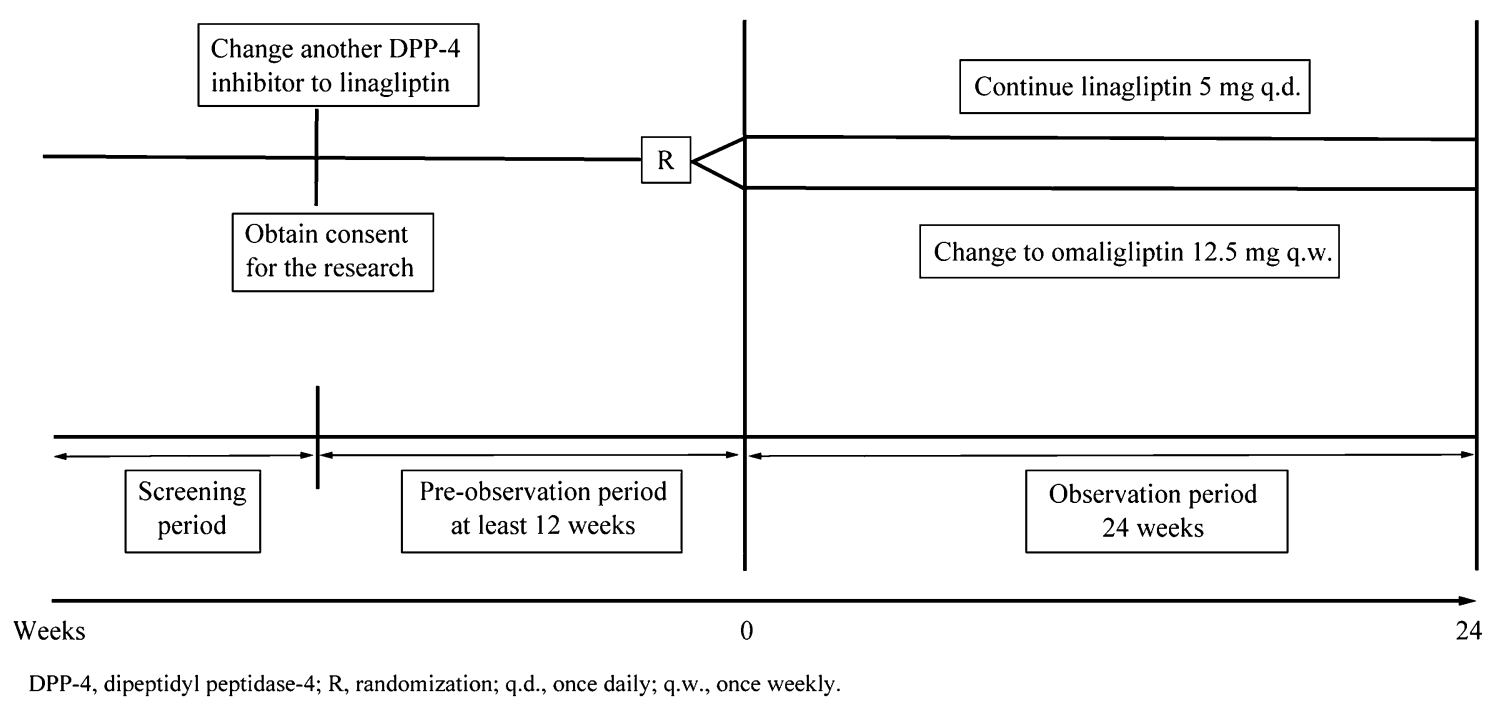

Fig. 1 Study design. DPP4 dipeptidyl peptidase 4, R randomization, q.d. once daily, q.w. once weekly

replacement or addition of OHAs was allowed until 12 weeks. After 12 weeks, if patients had not achieved $\mathrm{HbA} 1 \mathrm{c}<10 \%$ or $\mathrm{GA}<29 \%$, physicians were permitted to add other OHAs such as glinides or alpha-glucosidase inhibitors at their discretion in both treatment groups. However, additional use of insulin was not allowed. We did not restrict the use of therapeutic drugs for other conditions, such as antiplatelet, antihypertensive, or antidyslipidemia drugs.

\section{Study Evaluations}

The primary objectives of this study were to assess the efficacy, safety, and effect on quality of life (QOL) of once-weekly omarigliptin compared with once-daily linagliptin over 24 weeks of treatment. The hypothesis was that treatment with omarigliptin would provide a noninferior reduction in HbA1c and GA compared with linagliptin over the 24 weeks of treatment.

Primary endpoints were changes from baseline HbA1c and GA in both groups. Secondary endpoints were changes in blood glucose, plasma glucagon, and active GLP-1 before and after a single HD session and treatment satisfaction determined using the Diabetes TherapyRelated QOL (DTR-QOL) questionnaire score. Safety endpoints included incidence of adverse events due to omarigliptin, such as hypoglycemia.

\section{Laboratory Investigations}

Body mass index (BMI) was determined by dividing average body weight (kilograms) after a single HD session by height (meters) squared. Pre-HD venous blood samples were obtained at the beginning of the week. Routine biochemical parameters were analyzed in the laboratory at each facility. Samples were obtained for blood glucose, plasma glucagon, and plasma active GLP-1 measurements in the middle of the week. Then, measurements were performed at SRL, Inc. (Tokyo, Japan). Plasma glucagon levels were measured using a commercially available sandwich ELISA kit, which uses monoclonal antibodies against both the C-terminal and $\mathrm{N}$-terminal of glucagon (10-1271-01, Mercodia AB, Uppsala, Sweden). Plasma active GLP-1 levels were measured using a Glucagon-Like Peptide-1 (Active) ELISA Kit (Millipore, Billerica, MA).

Patient satisfaction was assessed using the 29-item, self-administered DTR-QOL questionnaire $[9,10]$. The DTR-QOL consists of four primary scales: domain 1 , burden on social activities and daily activities (13 items); domain 2, anxiety and dissatisfaction with 
treatment (8 items); domain 3, hypoglycemia (4 items); and domain 4 , satisfaction with treatment (4 items) $[9,10]$.

\section{Statistical Analysis}

The primary hypothesis of this study was that once-weekly omarigliptin was not significantly inferior to once-daily linagliptin in terms of glycemic control for patients with T2DM on maintenance HD. The recommended non-inferiority margin of $\mathrm{HbA1c}$ was $0.30-0.40 \%$, but we defined that value as $0.25 \%$ to show the noninferiority more precisely [11]. We also defined the non-inferiority margin of GA as $0.75 \%$, because the GA value was about three times the HbA1c value reported in a previous clinical study [12]. For sample size calculation, we used the non-inferiority margin of $\mathrm{HbA} 1 \mathrm{c}$ reduction. It was assumed that the $\mathrm{HbA} 1 \mathrm{c}$ reduction would be similar to that in previous clinical trials where omarigliptin resulted in an HbA1c decrease of $0.80 \%$ [6], whereas linagliptin resulted in a decrease of $0.87 \%$ [13], with a standard deviation (SD) of $0.16 \%$ for both treatments. With a non-inferiority margin of $0.25 \%$, one-sided alpha of 0.025 , and power of 0.8 , the sample size was calculated as 13 for each group. Considering a dropout rate of $10 \%$, the minimum sample size was set to 30 in total.

Efficacy analyses were performed for the full analysis set, which included participants who received an allocated treatment and provided assessable outcome data. Safety data were evaluated for all participants who received the allocated treatment at least once. Numerical variables were expressed as means $\pm \mathrm{SD}$, and categorical variables were expressed as $n$ (\%). Changes in all assessed numerical data during the 24-week study period were compared between the groups using the two-sample $t$ test, and two-sided $p$ values less than 0.05 were considered significant. For HbA1c and GA, the primary endpoints of this study, baseline-adjusted mean changes were also compared between groups on the basis of linear regression models in post hoc analyses. For the difference in each primary endpoint between the omarigliptin and linagliptin groups, the two-tailed
95\% confidence interval (CI) was calculated and the upper limit was used to evaluate the noninferiority. For HbA1c, Welch's $t$ test was used for unadjusted comparison and robust 95\% confidence intervals were calculated for both unadjusted and baseline-adjusted models because variances of mean changes were statistically different between the groups. All statistical analyses, except for the robust 95\% confidence interval for the baseline-adjusted model, were performed using IBM SPSS Statistics for Windows ver. 21.0 (IBM Corp., Armonk, NY). The robust 95\% interval was estimated using HAD, an Excel-based free statistical program package that can be downloaded via the internet (https://norimune.net/had, Japanese) [14].

\section{Compliance with Ethics Guidelines}

The study was approved by the institutional review boards of Niigata University and Shinrakuen Hospital. All procedures were performed in accordance with the Helsinki Declaration of 1964, and its later amendments, and conformed with national regulations. The study was a prospective randomized controlled trial, and all patients provided written informed consent for participation in this study and publication of their clinical data for research purposes.

\section{RESULTS}

The patient disposition is shown in Fig. 2. A total of 57 patients were screened and 24 were excluded (screening failure or rejection). A total of 33 patients were randomized, 17 to omarigliptin and 16 to linagliptin. Thirty patients received the study treatment. Three patients dropped out before starting treatment because of unexpected worsening of glycemic control, withdrawal of consent, or transfer to another hospital. None of patients in the omarigliptin group experienced hypoglycemia over the 24 weeks; one patient in the linagliptin group dropped out at week 21 because of hypoglycemia (about 50-60 mg/dL) during HD. This patient was not administered insulin or another antihyperglycemic agent. Two patients in the 


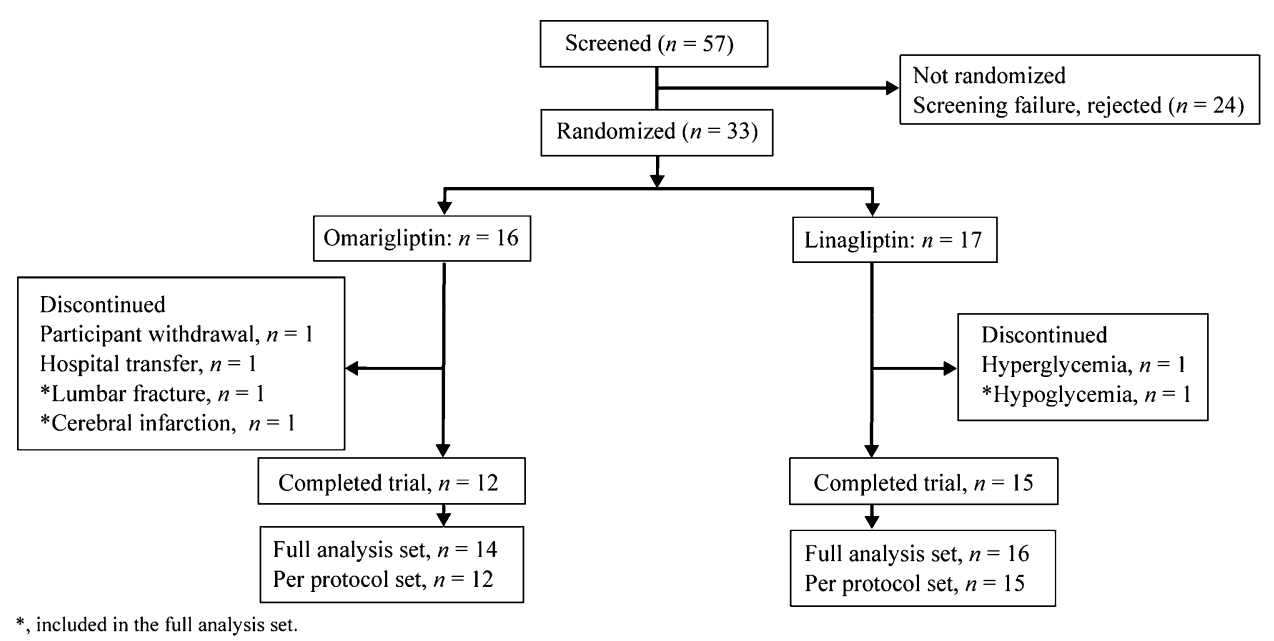

Fig. 2 Patient disposition. * Included in the full analysis set

omarigliptin group dropped out because of long-term hospitalization due to cerebral infarction or lumbar compression fracture. Their attending physicians deemed that there was no relationship between omarigliptin and these diseases. The full analysis set comprised 14 patients in the omarigliptin group and 16 patients in the linagliptin group, making a total of 30 patients (Fig. 2). The baseline clinical characteristics are shown in Table 1. Mean age was 67.6 years, approximately $80 \%$ were male, mean BMI was $23.5 \mathrm{~kg} / \mathrm{m}^{2}$, mean $\mathrm{HbA} 1 \mathrm{c}$ was $6.3 \%$, mean GA was $20.4 \%$, mean duration of maintenance HD was 6.1 years, and mean duration of T2DM was 18.8 years $(20.8 \pm 11.3$ in the linagliptin group; $16.0 \pm 8.7$ years in the omarigliptin group). Most of the patients were started on maintenance HD treatment because of diabetic nephropathy. For concomitant drugs, the mean number of tablets per week was 124.3. There were no changes in insulin doses and prescribed OHAs in either group during the study period.

The between-group differences in the changes in HbA1c and GA at 24 weeks are shown in Table 2 and Figs. 3 and 4 . In the full analysis set $(n=30)$, the differences in the mean change in primary endpoint values between the omarigliptin and linagliptin groups were $-0.61 \%$ $[-1.14,-0.09]$ for HbA1c, with a two-tailed upper $95 \%$ limit of $0.25 \%$, below the non-inferiority limit, and $-1.67 \%[-4.23,+0.88]$ for
GA, with a two-tailed upper $95 \%$ limit of $0.75 \%$, above the non-inferiority limit. In post hoc analyses adjusted for baseline values, the differences in the mean change between the omarigliptin and linagliptin groups were $0.62 \%[-1.20,-0.04]$ for HbA1c, with a twotailed upper $95 \%$ limit of $0.25 \%$, below the noninferiority limit, and $-2.49 \%[-5.15,+0.18]$ for GA, with a two-tailed upper $95 \%$ limit of $0.75 \%$, below the non-inferiority limit (Fig. S1 in the supplementary material). Also, HbA1c reduction was significantly greater in the omarigliptin group $(-0.2 \% \pm 0.6 \%)$ than in the linagliptin group $(0.4 \% \pm 0.8 \%, p=0.024)$. GA tended to be reduced in the omarigliptin group $(-0.3 \% \pm 3.4 \%)$ compared with the linagliptin group $(1.4 \% \pm 3.4 \% ; P=0.190)$.

The secondary endpoints are shown in Table 3 and Table S1. Blood glucose reduction after a single HD session was significantly greater in the omarigliptin group $(-18.4 \pm 31.4 \mathrm{mg} / \mathrm{dL})$ than in the linagliptin group $(25.2 \pm 59.5 \mathrm{mg} / \mathrm{dL}, p=0.019)$. Plasma glucagon levels before a single HD session tended to be reduced in the omarigliptin group compared with the linagliptin group $(-9.9 \pm 30.3 \mathrm{pg} / \mathrm{mL}$ and $6.1 \pm 31.6 \mathrm{pg} / \mathrm{mL}, p=$ 0.176). Plasma glucagon levels after a single HD session also tended to be reduced in the omarigliptin group compared with the linagliptin group $(-6.8 \pm 14.1 \mathrm{pg} / \mathrm{mL}$ and $2.6 \pm 16.6 \mathrm{pg} / \mathrm{mL}$, $p=0.109$ ). Plasma active GLP-1 levels before a 
Table 1 Baseline clinical characteristics

\begin{tabular}{|c|c|c|}
\hline & $\begin{array}{l}\text { Omarigliptin } \\
\text { group }\end{array}$ & $\begin{array}{l}\text { Linagliptin } \\
\text { group }\end{array}$ \\
\hline$n$ & 14 & 16 \\
\hline Age, years & $67.7 \pm 8.9$ & $67.5 \pm 9.0$ \\
\hline Male, $n(\%)$ & $12(85.7)$ & $12(75.0)$ \\
\hline $\begin{array}{l}\text { Duration of diabetes } \\
\text { (years) }\end{array}$ & $16.0 \pm 8.7$ & $20.8 \pm 11.3$ \\
\hline Duration of HD (years) & $5.6 \pm 4.9$ & $6.3 \pm 4.4$ \\
\hline BMI $\left(\mathrm{kg} / \mathrm{m}^{2}\right)$ & $23.5 \pm 2.7$ & $23.5 \pm 3.8$ \\
\hline HbAlc (\%) & $6.2 \pm 0.9$ & $6.5 \pm 1.0$ \\
\hline GA (\%) & $18.8 \pm 4.3$ & $21.9 \pm 3.8$ \\
\hline Hemoglobin $(\mathrm{g} / \mathrm{dL})$ & $10.5 \pm 1.2$ & $10.3 \pm 1.1$ \\
\hline Hematocrit (\%) & $31.9 \pm 3.9$ & $31.7 \pm 3.2$ \\
\hline Insulin use, $n(\%)$ & $1(7.1)$ & $3(18.5)$ \\
\hline Glinide use, $n(\%)$ & $0(0)$ & $3(18.5)$ \\
\hline$\alpha-G I$ use, $n(\%)$ & $0(0)$ & $3(18.5)$ \\
\hline $\begin{array}{l}\text { Other concomitant drugs } \\
\text { (tablets/week) }\end{array}$ & $121.1 \pm 81.2$ & $127.2 \pm 82.1$ \\
\hline \multicolumn{3}{|l|}{ Primary disease for ESRD } \\
\hline $\begin{array}{l}\text { Diabetic nephropathy, } \\
n(\%)\end{array}$ & $12(85.7)$ & $11(68.8)$ \\
\hline Nephrosclerosis, $n$ (\%) & $1(7.1)$ & $1(6.3)$ \\
\hline Other diseases, $n(\%)$ & $2(14.3)$ & $3(18.5)$ \\
\hline
\end{tabular}

Data are presented as mean \pm standard deviation, or $n(\%)$

$B M I$ body mass index, $H b A 1 c$ glycated hemoglobin, $G A$ glycoalbumin, $E S A$ erythropoiesis-stimulating agents, $H D$ hemodialysis, $\alpha$-GI $\alpha$-glucosidase inhibitors, ESRD endstage renal disease single $\mathrm{HD}$ session tended to be increased in the omarigliptin group compared with the linagliptin group $(7.6 \pm 12.5 \mathrm{pmol} / \mathrm{L}$ and $2.1 \pm 7.1$ pmol/L, $p=0.168)$. The mean change in the total DTR-QOL score was $-1.5 \pm 18.3$ in the omarigliptin group and $-3.0 \pm 10.2$ in the linagliptin group $(p=0.799)$. There were no significant differences in any subscale score of the DTR-QOL between the two groups.

There were no significant differences in changes of hemoglobin or hematocrit levels (Table S2). We also examined the doses of erythropoiesis-stimulating agents (ESAs) during the study period (Table S3). From the start to end of the observation period, the dose of ESAs increased in six patients, did not change in eight, and decreased in none in the omarigliptin group, whereas the dose increased in two patients, did not change in 11, and decreased in three in the linagliptin group.

There was also no change in the rate of insulin use or in the dialysis fluid glucose concentration between the two groups during the study period (data not shown).

\section{DISCUSSION}

This prospective, randomized, open-label, parallel-group, non-inferiority/superiority, multicenter study examined the efficacy of onceweekly DPP4 inhibitor omarigliptin compared with once-daily DPP4 inhibitor linagliptin in patients with T2DM undergoing maintenance HD. We confirmed the non-inferiority of omarigliptin compared with linagliptin in terms of changes in HbA1c. Moreover, we confirmed greater reduction in HbA1c and blood glucose after a single HD session in the omarigliptin

Table 2 Primary endpoints

\begin{tabular}{|c|c|c|c|c|c|c|c|c|c|}
\hline & \multicolumn{4}{|c|}{ Omarigliptin group } & \multicolumn{4}{|c|}{ Linagliptin group } & \multirow[t]{2}{*}{$p$ value } \\
\hline & $n$ & Week 0 & Week 24 & $\Delta 24$ weeks & $n$ & Week 0 & Week 24 & $\Delta 24$ weeks & \\
\hline HbAlc (\%) & 14 & $6.2 \pm 0.9$ & $6.0 \pm 0.6$ & $-0.2 \pm 0.6$ & 16 & $6.5 \pm 1.0$ & $6.9 \pm 1.5$ & $0.4 \pm 0.8$ & 0.024 \\
\hline GA (\%) & 14 & $18.8 \pm 4.3$ & $18.5 \pm 4.0$ & $-0.3 \pm 3.4$ & 16 & $21.9 \pm 3.8$ & $23.3 \pm 4.7$ & $1.4 \pm 3.4$ & 0.190 \\
\hline
\end{tabular}

Date are presented as mean \pm standard deviation. $\Delta 24$ weeks indicates changes from baseline to 24 weeks

$H b A 1 c$ glycated hemoglobin, $G A$ glycoalbumin 

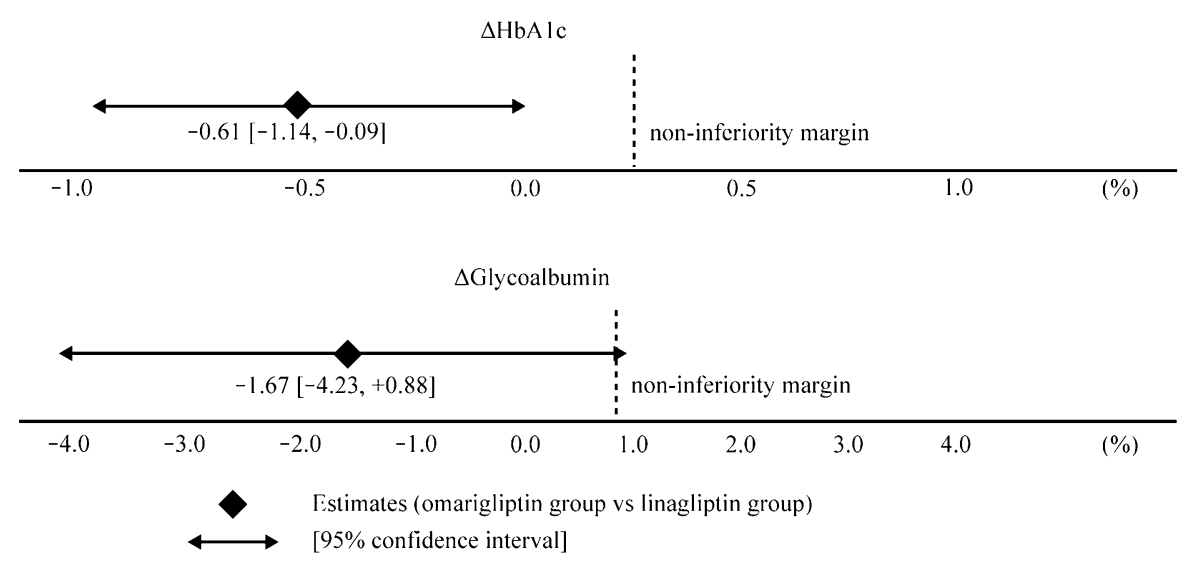

The vertical dotted line at $0.25 \%$ represents the margin of non-inferiority for the $\mathrm{HbAl} / \mathrm{c}$ change and $0.75 \%$ for the GA change.

Two-headed horizontal arrow bars represent one-sided $95 \%$ confidential intervals.

Fig. 3 Between-group differences in 24-week changes in glycated hemoglobin (HbAlc) and glycoalbumin (GA). The vertical dotted line at $0.25 \%$ represents the margin of non-inferiority for the HbAlc change and $0.75 \%$ for the GA change. Two-headed horizontal arrow bars represent one-sided $95 \%$ confidence intervals

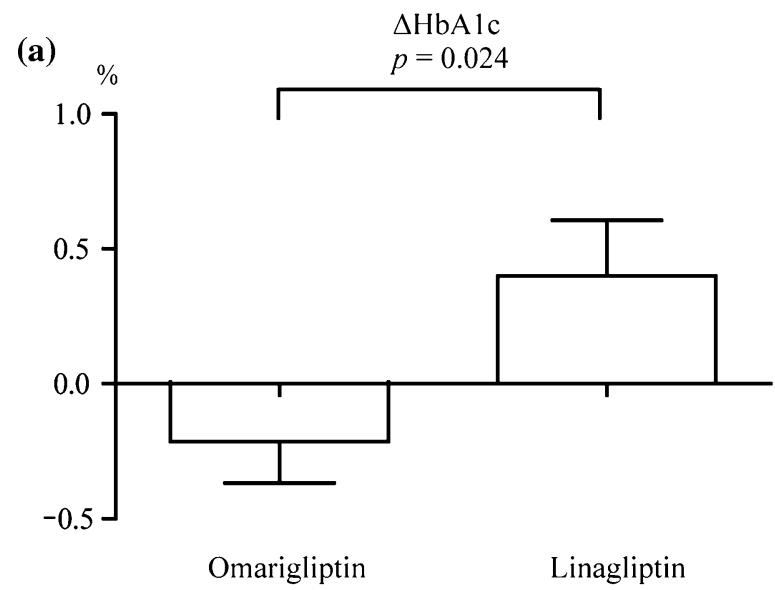

(b)

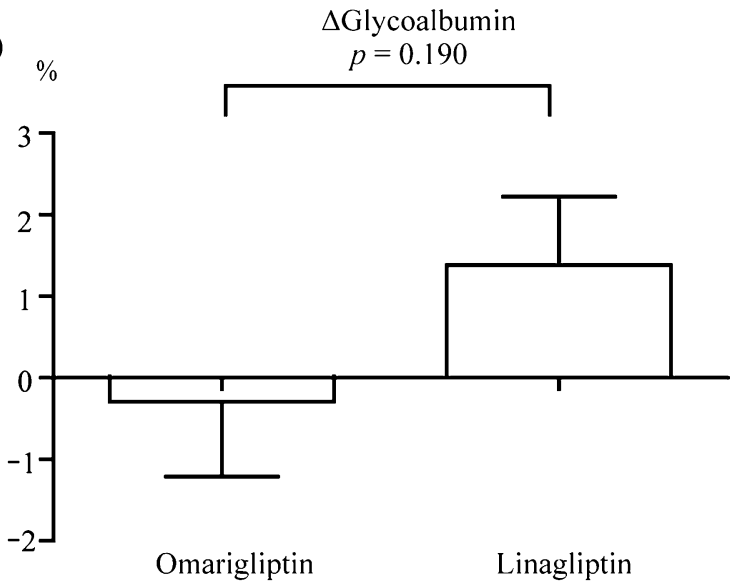

Changes in (a) HbAlc levels and (b) GA levels between the two groups. Between-group differences were compared using the t test. $P$ values $<0.05$ were considered statistically significant. Data are shown as the mean \pm standard error.

Fig. 4 Changes from baseline in glycated hemoglobin (HbAlc) and glycoalbumin (GA) after 24 weeks in the full analysis set. Changes in a HbAlc levels and b GA levels between the two groups. Between-group differences were

group. None of the patients in the omarigliptin group developed hypoglycemia during the observation period.

Diabetic nephropathy is the leading cause of end-stage renal disease (ESRD) worldwide. Good glycemic control is reported to improve prognosis even in patients with T2DM on maintenance $\mathrm{HD}$ [2], but few OHAs can be used in such patients, so insulin injection therapy is the compared using the $t$ test. $p$ values less than 0.05 were considered statistically significant. Data are shown as the mean \pm standard error

standard. However, adherence to insulin injection therapy is usually lower than adherence to OHAs because of hypoglycemia. DPP4 inhibitors are widely used in patients with T2DM on maintenance HD and have a good safety profile. For example, vildagliptin, a once-daily DPP4 inhibitor, also reduces plasma glucagon levels and might contribute to reduction of blood glucose in patients on maintenance HD [15]. 
Table 3 Secondary endpoints

\begin{tabular}{|c|c|c|c|c|c|}
\hline & \multicolumn{2}{|c|}{ Omarigliptin group } & \multicolumn{2}{|c|}{ Linagliptin group } & \multirow[t]{2}{*}{$p$ value } \\
\hline & $\bar{n}$ & $\Delta 24$ weeks & $n$ & $\Delta 24$ weeks & \\
\hline Glucose before $\mathrm{HD}(\mathrm{mg} / \mathrm{dL})$ & 13 & $1.2 \pm 56.8$ & 16 & $45.1 \pm 91.8$ & 0.127 \\
\hline Glucose after HD (mg/dL) & 13 & $-18.4 \pm 31.4$ & 16 & $25.2 \pm 59.5$ & 0.019 \\
\hline Glucagon before $\mathrm{HD}(\mathrm{pg} / \mathrm{mL})$ & 13 & $-9.9 \pm 30.3$ & 16 & $6.1 \pm 31.6$ & 0.176 \\
\hline Glucagon after $\mathrm{HD}(\mathrm{pg} / \mathrm{mL})$ & 13 & $-6.8 \pm 14.1$ & 16 & $2.6 \pm 16.6$ & 0.109 \\
\hline Active GLP-1 before HD (pmol/L) & 13 & $7.6 \pm 12.5$ & 16 & $2.1 \pm 7.1$ & 0.168 \\
\hline Active GLP-1 after HD (pmol/L) & 13 & $-3.0 \pm 13.0$ & 16 & $-0.7 \pm 10.5$ & 0.610 \\
\hline \multicolumn{6}{|l|}{ DTR-QOL } \\
\hline Total score & 13 & $-1.5 \pm 18.3$ & 16 & $-3.0 \pm 10.2$ & 0.799 \\
\hline \multicolumn{6}{|l|}{ Subscale score } \\
\hline Domain 1 & 13 & $-2.6 \pm 23.5$ & 16 & $-2.9 \pm 14.6$ & 0.966 \\
\hline Domain 2 & 13 & $1.1 \pm 15.5$ & 16 & $-3.7 \pm 12.1$ & 0.374 \\
\hline Domain 3 & 13 & $-3.8 \pm 17.5$ & 16 & $-5.8 \pm 26.0$ & 0.809 \\
\hline Domain 4 & 13 & $-0.5 \pm 31.1$ & 16 & $-0.5 \pm 17.6$ & 0.997 \\
\hline
\end{tabular}

Data are presented as mean \pm standard deviation. $\triangle 24$ weeks indicates changes from baseline to 24 weeks $H D$ hemodialysis, GLP-1 glucagon-like peptide-1, GIP gastric inhibitory polypeptide, DTR-QOL Diabetes Therapy-Related Quality of Life

Moreover, it was reported that patients with ESRD including those on peritoneal dialysis with T2DM showed no significant difference in glycemic control among three types of oncedaily DPP4 inhibitors [16]. Once-weekly DPP4 inhibitor omarigliptin showed non-inferiority to other OHAs in improving glycemic control and might thus improve adherence and patient satisfaction [17]. In a meta-analysis, omarigliptin showed obviously better efficacy and safety and lower risk of hypoglycemia than placebo [5]. Also, omarigliptin can be used at low doses in patients with ESRD and is favorably comparable with placebo or glipizide in terms of efficacy and safety [18]. In our study, we found that patients in the once-weekly omarigliptin group had greater reduction in $\mathrm{HbA1c}$ and blood glucose after a single HD session compared with patients in the once-daily DPP4 inhibitor linagliptin group. Although this was not a significant difference, we also found that the omarigliptin group had reduced plasma glucagon and active GLP-1 levels either before or after a single HD session compared with the linagliptin group. There are two possible reasons for the better glycemic control in the omarigliptin group. First, omarigliptin is a longacting OHA, so this drug can maintain higher DPP4 inhibition over the period of a week [19]. Plasma glucagon levels also tended to be reduced in the omarigliptin group in our study. Furthermore, it has been reported that omarigliptin might decrease DPP4 secretion and ameliorate insulin resistance compared with linagliptin [20]. Accordingly, treatment with omarigliptin might have more strongly suppressed plasma glucagon and increased active GLP-1 than treatment with linagliptin over the period of a week, although more detailed studies are needed. Second, once-weekly drugs, such as omarigliptin, might improve adherence by reducing the medication burden of patients. Some once-weekly DPP4 inhibitors are currently available for use. Inagaki et al. reported that the once-weekly DPP4 inhibitor trelagliptin, which is contraindicated in patients with ESRD, 
provides well-tolerated long-term safety and efficacy; therefore, once-weekly drugs are considered a good therapeutic alternative in patients with T2DM for improving adherence $[21,22]$. The same as in our study, if patients undergoing HD can take once-weekly drugs at the HD center, almost perfect adherence might be possible. It also might be easy for patients to take once-weekly DPP4 inhibitors at the HD center because they are usually also administered other once-weekly drugs such as ESAs.

Some studies have reported improved treatment satisfaction when patients with T2DM switched from once-daily to once-weekly drugs, based on the Diabetes Treatment Satisfaction Questionnaire [23]. The DTR-QOL instrument that we used is an exact reflection of the patient's satisfaction with treatment, especially in relatively younger patients under the age of 65 years [24], those receiving at most two concomitant drugs for treatment of comorbidities, and those who were treatment naïve before the start of the study [25]. As such, we expected some improvement in patient satisfaction with treatment, but there was no significant improvement in DTR-QOL score in the omarigliptin group. In our study, the participants were relatively old (mean age 67.6 years). They were also not treatment naïve and received many concomitant drugs. The mean number of concomitant drug types was 8.0 per day (17.9 tablets per day, 124.3 tablets per week). In general, patients on maintenance HD require many drugs, including antihypertensives or phosphorus binders. These factors might explain the lack of significant improvement in DTR-QOL score in our study. Among those patients who received hundreds of tablets per week, treatment satisfaction did not improve even when the number of tablets decreased by six tablets per week.

In Japan, rising medical costs for patients with T2DM on $\mathrm{HD}$ are a major challenge. Monthly medical expenses for maintenance HD are estimated at about 400,000 JPY per patient. Kanozawa et al. reported that switching from other DPP4 inhibitors to lower-dose sitagliptin on the basis of the patient's renal function status reduced daily drug costs by 88.1 JPY per patient [26]. Omarigliptin (12.5 mg), as used in this study, costs about 492.9 JPY weekly, and linagliptin (5 mg) costs 1005.2 JPY weekly. Switching linagliptin to omarigliptin reduces drug costs per patient by 512.3 JPY weekly and about 30,000 JPY yearly. Notably, the daily DPP4 inhibitor sitagliptin $(12.5 \mathrm{mg}$ dose in patients on hemodialysis), which is widely used worldwide, costs about $400 \mathrm{JPY}$ weekly, which is comparable to the cost of omarigliptin. Omarigliptin can therefore be a good therapeutic option in terms of reducing national medical costs.

This study has several limitations. First, although we confirmed that the omarigliptin group had a greater reduction in HbA1c, this result might have been affected by changes in the doses of ESAs in the two groups. Second, we could not confirm non-inferiority of omarigliptin compared with linagliptin in reduction of GA levels in unadjusted data. We calculated the sample size of participants based on the non-inferiority margin in HbA1c. As a result, the sample size may have been too small to identify a significant difference in the reduction of GA levels between the two groups. Setting a larger sample size might help to confirm noninferiority in terms of not only reduction in GA but also reduction in plasma glucagon and elevation in plasma active GLP-1 levels, so we will consider using a crossover protocol in a future study. Third, most of our participants already had good glycemic control when this study started. For ethical reasons, we excluded the patients with poor glycemic control (HbA1c $\geq 9 \%$ or $\mathrm{GA} \geq 27 \%$ ) on current treatment because they had the potential for further exacerbation of glycemic control because of drug changes. Investigating the efficacy of omarigliptin in patients with poor glycemic control remains a topic for future research. Fourth, because of ethical considerations, our protocol permitted patients to change or add OHAs if they had not achieved HbA1c $<10 \%$ or GA $<29 \%$ after 12 weeks. However, no patients changed or added antidiabetic drugs during the study period, so this point did not appear to affect our results. Fifth, mean duration of diabetes was longer in the linagliptin group than in the omarigliptin group. This difference might have affected our results, but the sample size in 
this study was too small to perform multivariable analysis. This point would need to be adjusted for in a further study. Sixth, we could not measure morning fasting plasma glucagon or active GLP-1 because some of our patients underwent HD at night. Seventh, the study period was only 24 weeks, so the efficacy, safety, and treatment satisfaction of omarigliptin over longer periods remain unclear. Eighth, we compared only linagliptin and omarigliptin, and therefore the effects of omarigliptin in comparison with other OHAs are not known.

\section{CONCLUSION}

Our data showed that the once-weekly DPP4 inhibitor omarigliptin was non-inferior to oncedaily DPP4 inhibitor linagliptin in glycemic control. Use of once-weekly omarigliptin is also practicable for achieving glycemic control in patients with T2DM on maintenance HD. Further research is needed to verify the effectiveness of omarigliptin in longer-term and largerscale studies.

\section{ACKNOWLEDGEMENTS}

Funding. No funding or sponsorship was received for this study. The journal's Rapid Service Fee was paid by Niigata University.

Authorship. All named authors meet the International Committee of Medical Journal Editors (ICMJE) criteria for authorship of this article, take responsibility for the integrity of the work as a whole, and have given their approval for this version to be published.

Authorship Contributions. Y. Yoshizawa, M. Hosojima, and H. Kabasawa were responsible for the conception and design of the study. Y. Yoshizawa was the chief investigator and also responsible for data analysis. M. Hosojima, $\mathrm{H}$. Kabasawa, and N. Tanabe were responsible for data analysis. Y. Yoshizawa, M. Hosojima, and $\mathrm{H}$. Kabasawa were responsible for data acquisition. Y. Yoshizawa, M. Hosojima, H. Kabasawa,
N. Tanabe, D. Ugamura, Y. Koda, H. Shimada, T. Takasawa, T. Ito, T. Kitamura, M. Kobayashi, Y. Suzuki, A. Saito, and I. Narita were responsible for data interpretation. Y. Yoshizawa and M. Hosojima were responsible for drafting the manuscript. All authors contributed to writing the final manuscript.

Medical Writing and Editorial Assistance. The authors thank Dr. Hitoshi Ishii (Department of Diabetology, Nara Medical University) for advice on the DTR-QOL. The authors also thank Ms. Maiko Daisaka at Niigata University for providing technical assistance and ThinkSCIENCE Inc. (Tokyo, Japan) for providing language editing services. This assistance was paid for by Niigata University.

Prior Presentation. Data included in this manuscript were presented, in part, at the American Diabetes Association 79th Scientific Sessions 2019 in San Francisco, California.

Disclosures. Michihiro Hosojima, Hideyuki Kabasawa, Tadahiro Kitamura, Akihiko Saito, and Ichiei Narita have received lecture fees from Eli Lilly Japan K.K., MSD K.K., and Nippon Boehringer Ingelheim Co., Ltd.; Yutaka Koda has received lecture fees from MSD K.K.; Michihiro Hosojima has received research support from Eli Lilly Japan K.K. and MSD K.K.; Akihiko Saito and Ichiei Narita have received research support from Eli Lilly Japan K.K., MSD K.K., and Nippon Boehringer Ingelheim Co., Ltd. Yuta Yoshizawa, Naohito Tanabe, Daisuke Ugamura, Hisaki Shimada, Tetsuya Takasawa, Takahito Ito, Masaki Kobayashi and Yoshiki Suzuki have nothing to disclose.

Compliance with Ethics Guidelines. The study was approved by the institutional review boards of Niigata University and Shinrakuen Hospital. All procedures were performed in accordance with the Helsinki Declaration of 1964, and its later amendments, and conformed with national regulations. The study was a prospective randomized controlled trial, and all patients provided written informed consent for participation in this study and publication of their clinical data for research purposes. 
Data Availability. The datasets generated during and/or analyzed during the current study are available from the corresponding author on reasonable request.

Open Access. This article is licensed under a Creative Commons Attribution-NonCommercial 4.0 International License, which permits any non-commercial use, sharing, adaptation, distribution and reproduction in any medium or format, as long as you give appropriate credit to the original author(s) and the source, provide a link to the Creative Commons licence, and indicate if changes were made. The images or other third party material in this article are included in the article's Creative Commons licence, unless indicated otherwise in a credit line to the material. If material is not included in the article's Creative Commons licence and your intended use is not permitted by statutory regulation or exceeds the permitted use, you will need to obtain permission directly from the copyright holder. To view a copy of this licence, visit http://creativecommons.org/licenses/by$\mathrm{nc} / 4.0 /$.

\section{REFERENCES}

1. Nathan DM, Genuth S, Lachin J, et al. The effect of intensive treatment of diabetes on the development and progression of long-term complications in insulin-dependent diabetes mellitus. N Engl J Med. 1993;329(14):977-86.

2. Nakamura Y, Hasegawa H, Tsuji M, et al. Diabetes therapies in hemodialysis patients: dipeptidase- 4 inhibitors. World J Diabetes. 2015;6(6):840-9.

3. Lo C, Toyama T, Wang Y, et al. Insulin and glucoselowering agents for treating people with diabetes and chronic kidney disease. Cochrane Database Syst Rev. 2018;9:CD011798.

4. Meguro S, Matsui S, Itoh H. Treatment preference for weekly versus daily DPP-4 inhibitors in patients with type 2 diabetes mellitus: outcomes from the TRINITY trial. Curr Med Res Opin. 2019;35(12): 2071-8.

5. Wang X, Li X, Qie S, Zheng Y, Liu Y, Liu G. The efficacy and safety of once-weekly DPP-4 inhibitor omarigliptin in patients with type 2 diabetes mellitus: a systemic review and meta-analysis. Medicine (Baltimore). 2018;97(34):e11946.

6. Omarigliptin [product label]. MSD K.K. Tokyo, Japan. 2018. http://www.pmda.go.jp/PmdaSearch/ iyakuDetail/ResultDataSetPDF/170050_3969025F10 22_1_08 (Japanese). Accessed 2 Dec 2019.

7. Kothawala P, Badamgarav E, Ryu S, Miller RM, Halbert RJ. Systematic review and meta-analysis of real-world adherence to drug therapy for osteoporosis. Mayo Clin Proc. 2007;82(12):1493-501.

8. Lee A, Song X, Khan I, et al. Association of cinacalcet adherence and costs in patients on dialysis. J Med Econ. 2011;14(6):798-804.

9. Ishii H. Development and psychometric validation of the diabetes therapy-related QOL (DTR-QOL) questionnaire. J Med Econ. 2012;15(3):556-63.

10. Mita T, Hiyoshi $\mathrm{T}$, Yoshii $\mathrm{H}$, et al. The effect of linagliptin versus metformin treatment-related quality of life in patients with type 2 diabetes mellitus. Diabetes Ther. 2019;10(1):119-34.

11. Guidance for industry diabetes mellitus: developing drugs and therapeutic biologics for treatment and prevention. US Department of Health and Human Services Food and Drug Administration Center for Drug Evaluation and Research (CDER): US FDA; February, 2008. https://www.fda.gov/media/71289/ download. Accessed 10 Feb 2020.

12. Tsuruta Y, Ichikawa A, Kikuchi K, Echida Y, Onuki T, Kosaku Nitta K. Glycated albumin is a better indicator of the glucose excursion than predialysis glucose and hemoglobin A1c in hemodialysis patients. Renal Replace Ther. 2016;2(3). https://doi. org/10.1186/s41100-016-0013-7.

13. Linagliptin [product label]. Nippon Boehringer lngelheim. 2018. http://www.pmda.go.jp/Pmda Search/iyakuDetail/ResultDataSetPDF/650168_3969 014F1024_1_17 (Japanese). Accessed 2 Dec 2019.

14. Shimizu H. An introduction to the statistical free software HAD: suggestions to improve teaching, learning and practice data analysis. J Media Inf Commun. 2016;1:59-73.

15. Kume S, Uzu T, Takagi C, et al. Efficacy and tolerability of vildagliptin in type 2 diabetic patients on hemodialysis. J Diabetes Investig. 2012;3(3): 298-301.

16. Park SH, Nam JY, Han E, et al. Efficacy of different dipeptidyl peptidase-4 (DPP-4) inhibitors on metabolic parameters in patients with type 2 diabetes undergoing dialysis. Medicine (Baltimore). 2016;95(32):e4543. 
17. Tsuchiya S, Friedman E, Addy C, et al. Single and multiple dose pharmacokinetics and pharmacodynamics of omarigliptin, a novel, once-weekly dipeptidyl peptidase- 4 inhibitor, in healthy Japanese men. J Diabetes Investig. 2017;8(1):84-92.

18. Chacra A, Gantz I, Mendizabal G, et al. A randomised, double-blind, trial of the safety and efficacy of omarigliptin (a once-weekly DPP-4 inhibitor) in subjects with type 2 diabetes and renal impairment. Int J Clin Pract. 2017;71(6):e12955.

19. Jain L, Chain ASY, Tatosian DA, et al. Pharmacokinetic-pharmacodynamic (dipeptidyl peptidase-4 inhibition) model to support dose rationale in diabetes patients, including those with renal impairment, for once-weekly administered omarigliptin. Br J Clin Pharmacol. 2019;85(12):2759-71.

20. Hattori S. Omarigliptin decreases inflammation and insulin resistance in a pleiotropic manner in patients with type 2 diabetes. Diabetol Metab Syndr. 2020;12:24.

21. Inagaki N, Sano H, Seki Y, Kuroda S, Kaku K. Longterm safety and efficacy of a novel once-weekly oral trelagliptin as monotherapy or in combination with an existing oral antidiabetic drug in patients with type 2 diabetes mellitus: a 52-week open-label, phase 3 study. J Diabetes Investig. 2016;7(5): 718-26.
22. Inagaki N, Sano H, Seki Y, Kuroda S, Kaku K. Efficacy and safety of once-weekly oral trelagliptin switched from once-daily dipeptidyl peptidase- 4 inhibitor in patients with type 2 diabetes mellitus: an open-label, phase 3 exploratory study. J Diabetes Investig. 2018;9(2):354-9.

23. Tosaki T, Kamiya H, Yamamoto Y, et al. Efficacy and patient satisfaction of the weekly DPP-4 inhibitors trelagliptin and omarigliptin in 80 Japanese patients with type 2 diabetes. Intern Med. 2017;56(19):2563-9.

24. Suzuki K, Hasegawa K, Watanabe M. Efficacy and patient satisfaction of dipeptidyl peptidase- 4 inhibitor after switching from once-daily DPP-4 inhibitor to once-weekly regimen. J Clin Med Res. 2018;10(8):641-7.

25. Oita M, Miyoshi H, Ono K, et al. Satisfaction and efficacy of switching from daily dipeptidyl peptidase- 4 inhibitors to weekly trelagliptin in patients with type 2 diabetes-randomized controlled study. Endocr J. 2018;65(2):141-50.

26. Kanozawa K, Noguchi Y, Sugahara S, et al. The renoprotective effect and safety of a DPP-4 inhibitor, sitagliptin, at a small dose in type 2 diabetic patients with a renal dysfunction when changed from other DPP-4 inhibitors: REAL trial. Clin Exp Nephrol. 2018;22(4):825-34. 